\title{
OPTIMIZATION OF RFID SYSTEM COVERAGE IN A MANUFACTURING ENVIRONMENT
}

\author{
A Thesis \\ presented to \\ the Faculty of California Polytechnic State University, \\ San Luis Obispo
}

\author{
In Partial Fulfillment \\ of the Requirements for the Degree \\ Master of Science in Industrial Engineering
}

by

Scott Malatesta

June 2016 
(C) 2016

Scott Malatesta

ALL RIGHTS RESERVED 


\title{
COMMITTEE MEMBERSHIP
}

TITLE: Optimization Of RFID System Coverage

In A Manufacturing Environment

\author{
AUTHOR: $\quad$ Scott Malatesta
}

DATE SUBMITTED: June 2016

COMMITEE CHAIR: $\quad$ Tali Freed, Ph.D.

Professor of Industrial Engineering

COMMITEE MEMBER: $\quad$ Kenneth Doerr, Ph.D.

Associate Professor at the Navel Postgraduate

COMMITTEE MEMBER: $\quad$ Lawrence Sze, Ph.D.

Professor of Mathematics 


\begin{abstract}
Optimization Of RFID System Coverage In A Manufacturing Environment Scott Malatesta
\end{abstract}

This paper compiles existing ideas, theories, and experiments across multiple disciplines to provide guidance for a company looking to implement an optimal RFID system in their production facility. The desire is to maximize the information received by the system while minimizing the cost. Four potential layouts of RFID antennas, two with overlapping antenna coverage and two with non-overlapping layouts, are first analyzed to understand the special coverage and the number of antennas required. The value of information is then quantified to determine whether higher coverage layouts are worth the additional costs associated with the higher number of antennas required. It was found that the non-overlapping network of antennas in a hexagonal lattice formation, which covers $90.7 \%$ of the production floor area, provides the highest amount of benefit when considering the system's implementation costs. However, some companies prefer or even require a system that provides perfect information throughout the production process, so implementing an overlapping network may be considered optimal for some companies, even with the higher up front costs. 


\section{TABLE OF CONTENTS}

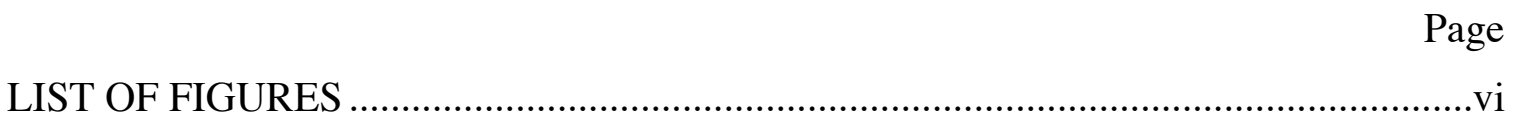

Chapter 1: Introduction and Problem Statement ...........................................................

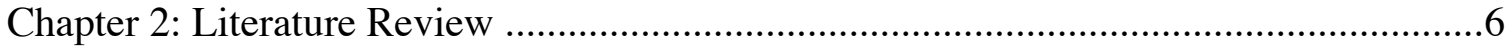

2.1: Research in the Field of Wireless Communication .........................................6

2.2: Research in RFID Coverage Optimization ................................................... 6

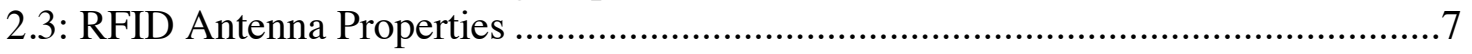

Chapter 3: Optimal Production Coverage Methods .......................................................9

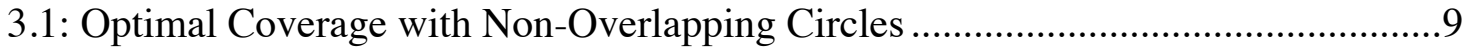

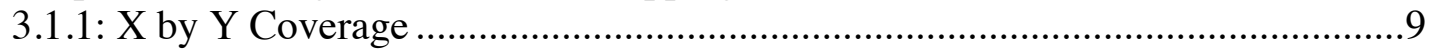

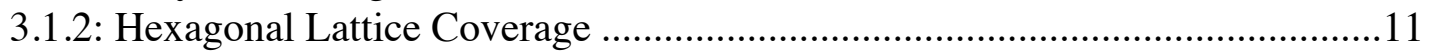

3.2: Optimization of Complete Floor Coverage Models ...........................................12

3.2.1: Optimal Overlapping of Circles ............................................................ 12

3.2.2: Square Grid RFID Reader Antenna Network ............................................13

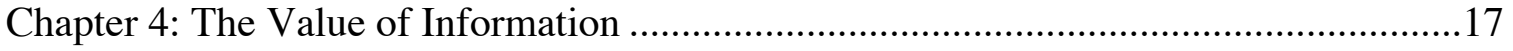

4.1: Method of Quantifying the Value of Information .............................................17

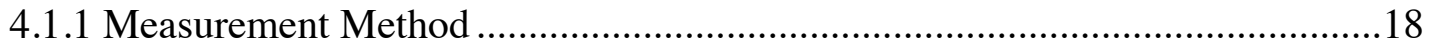

4.1.1.1: Location Estimation ..........................................................................19

4.1.1.2: Search Decisions Based on Available Actions....................................22

4.1.1.3: Calculating The Value of Information .............................................23

4.1.2: Determining the Impact of Missing Information ......................................25

4.1.3: Analysis Based on Quantitative Model .......................................................28

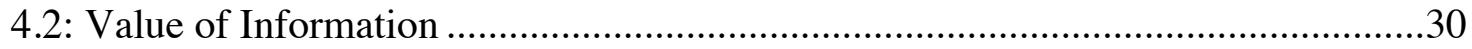

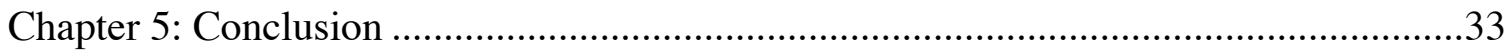

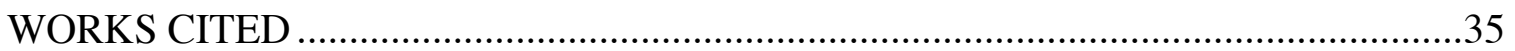




\section{LIST OF FIGURES}

Figure $\quad$ Page

Figure 1: The Hexagonal Lattice Formation (Anusha, 2005) ......................................11

Figure 2: $120.9 \%$ area of the circles is the minimum area required to cover the entire

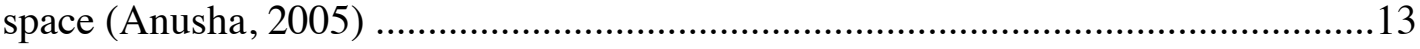

Figure 3: Square Grid Method Sample Layout (Tan et al., 2008) ..................................14

Figure 4: Four Overlapping Sections in Square Grid ............................................15

Figure 5: Accessing the Value of Information (Kelepouris \& McFarlane, 2010)............19

Figure 6: Distribution of Uncertainty (Kelepouris \& McFarlane, 2010) .........................26

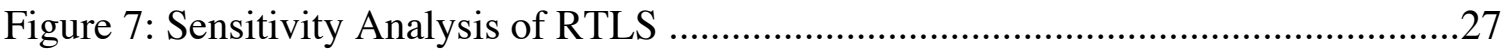

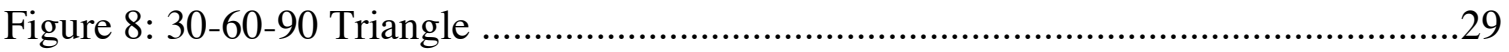




\section{Chapter 1: Introduction and Problem Statement}

Over the past few decades, a number of inventory management techniques have been invented to gain greater insight into the production process. One of the first methods for tracking objects in a system was the Active Badge System, developed in 1989 by Olivetti Research Laboratory. This system was created to help track people using badges on their bodies that released infrared signals to be read by a network sensor. Though the goal was to eventually expand this technology to inventory tracking, it was not expanded due to line-of-sight requirements and short-range signal transmission. Researchers then focused on other solutions such as ultrasound technology. However, in the last decade, the use of radio frequency technology has become the industry standard real-time location tracking system (RTLS), providing individuals with information on their inventory, tools, or other objects, in turn helping them to optimize the production process through the reduction of time spent searching for these objects (Patil et al., 2008).

Radio Frequency Identification (RFID) is a system that wirelessly transmits unique identities placed on objects or individuals utilizing radio waves. Unlike other forms of auto-IDs, such as more commonly known barcodes, RFID tags can contain other information that can be utilized by the user such as production date, sell by date, shipment date, etc. All of this information, as well as the ability to have up-to-the-minute knowledge of the location of specific items, provides the opportunity to have "perfect supply chain visibility," a common goal within many business strategies, particularly those that focus on just-in-time manufacturing (Violino, 2005). 
A RFID system is made up of readers or integrators, and transponders or tags. The reader sends radio transmissions to the tags that then returns information about the tagged object such as its location. This information is then sent by the reader to a network where it is added into a database or utilized by the user. These systems are used throughout the world in many different applications such as displaying the location of airplanes, tracking cars through toll booths to minimize traffic delays or to charge drivers tolls without forcing them to stop, identifying railcars that travel thousands of miles containing valuable goods and even identifying livestock where animals such as cows are tagged in their ear and tracked for any recalls if there have any diseases or health issues (Dobkin, 2007). These are just a few of many applications of RFID technology. However, this paper will focus on its use in production facilities.

There are many types of RFID technologies for different applications, each with a different combination of the radio frequency band used for communication and the way the tag is powered.

The electric field of a reader oscillates at different frequencies, measured in Hertz $(\mathrm{Hz})$, as electromagnetic waves travel at a constant rate. The frequencies of the reader's electric fields range from a few thousand to 100 billion $\mathrm{Hz}$. These different frequencies change the characteristics of the wavelengths and affect the interaction between the wavelengths and the tags.

Most RFID applications use one of four frequency bands ranging from $130 \mathrm{kHZ}$ to $2.4 \mathrm{GHz}$. The $130 \mathrm{kHz}$ band, or low-frequency band (LF), has a small wavelength and is relatively unaffected by the presence of water. This allows for the technology to be used in applications concerning livestock and humans. The reader antennas and tags are 
much smaller than the wavelengths, causing them to be inductively coupled with the magnetic fields from the reader antenna that are inducing voltages on the tag antenna. The read range for these LF tags is approximately the same size as the reader antenna, which is between a few centimeters and a meter. The movement of data from these LF tags is relatively slow, however its low cost is one of its greatest benefits.

The $13.56 \mathrm{MHz}$, or high-frequency $(\mathrm{HF})$, band is frequently utilized in industrial and medical applications. The wavelength, close to 20 meters, is also larger than the readers and tags and requires inductive coupling; however this band can move data at a much faster rate than LF bands, helping to shorten read time. This technology is often used in RFID-enabled credit cards.

There are also RFID systems that use bands near $900 \mathrm{MHz}$ and $2.4 \mathrm{GHz}$, operating in the ultra-high-frequency (UHF) range. The wavelengths in this range are considerably smaller, varying from one meter to ten centimeters. This size is more similar to the size of the tags and readers, allowing for a wider spread. These tags and readers are often radioactively coupled enabling read ranges from a few meters to kilometers. However, there can be interference between readers and other radio devices in the same frequency band. These tags move data incredibly quickly, transferring more data at a faster rate than the other frequency bands. UHF systems are often used for supply chain tracking at a non-itemized level such as pallets, or for asset tracking.

RFID tags must be powered to allow them to communicate with the readers. The three main power methods are classified into passive, semi-passive, and active tags.

Passive tags are the simplest. It receives their power from radio signals sent by the reader. These tags don't have a radio transmitter and don't create their own signal. The 
tags vary the electrical load on the antenna to alter the signal reflected, a practice known as backscatter communications. Due to their simplicity, passive tags are the cheapest option, sold for around 10 cents each. These tags require no maintenance and last as long as the tag's plastic material can hold up. One downfall of the passive tag is the large radio signals they require to be activated This limits the read ranges to a few centimeters to around 20 meters.

Semi-passive tags also use backscattering, however a battery is used to power the tags, helping to increase the read range. The received signal is proportional to $1 /$ reader tag distance^4, which quickly limits the read range, but still reads in the 100 meter range. These readers also provide higher reliability and are less affected by obstacles. Toll transmitters often use this technology to read cars coming through at higher speeds, even if they are covered by water from rain. These tags are more expensive than passive RFIDs, costing around $\$ 2$ per and requiring battery replacements over time.

In contrast, active tags have their own transmitter, which allows for read ranges up to kilometers even with obstructions. Active tags are often paired with UHF bands because inductively coupled systems can decrease this read range. These tags range in price from $\$ 20-\$ 100$ and are used to track expensive objects including shipping containers or trucks (Dobkin, 2007).

Cost is the major obstacle that has led to a slow implementation of RFID technology, with many companies not understanding the value these systems can provide. In 1999, the Auto-ID Center was created to continue research on the issue of cost, particularly on the costs for tags, which are often the largest cost over the lifetime of the system. With tags costing around 10-40 cents per unit, and with the implementation of 
the system adding costs, many companies have been hesitant to implement RFID technology in their manufacturing facilities (Violino, 2005).

In an attempt to create "perfect supply chain visibility," RFID technology has provided companies with the opportunity to access real time information from supplier to customer. While the entire supply chain benefits from the use of RFID tracking, manufacturing can utilize the technology to optimize everyday processes, such as helping to reduce costs and increase output. This can be achieved by helping to locate particular products or equipment throughout the manufacturing process; reducing search time and labor costs; leveraging real-time data to gain statistical insight into the production process for cost effective, data-backed, improvements; and decreasing product scrap through improved traceability.

However, with these benefits considered, the implementation, usage, and maintenance costs of RFID technology have proven to be an issue for many companies. This paper will look further into possible reduction of implementation and maintenance costs, focusing on minimizing the number of RFID antennas and readers.

Many methods can be used to optimize the layout of antennas on a production floor. Each provide advantages and disadvantages at different costs that need to be analyzed by management before the implementation of an RFID system that meets their particular needs.

The scope of this paper focuses on different theories and methods of production floor coverage by circularly polarized, ceiling-mounted RFID reader antennas. All items on this production floor are assumed to travel at a similar height from the ceiling throughout the entire production process. 


\section{Chapter 2: Literature Review}

\section{1: Research in the Field of Wireless Communication}

Studies related to the optimal coverage of RF towers have been conducted since the early 2000s due to the increase in global cell phone usage. In 2002, Adickes et al. proposed a methodology that identified the optimal number of receivers and their location by leveraging genetic algorithms and simulation to generate new positional locations until one solution is ultimately selected. In 2004, Huang et al. suggested a polynomial algorithm that ensures that every point in a given space is covered by at least $\alpha$ sensors, where $\alpha$ is an integer determined by the individual seeking optimization. For RFID systems, $\alpha$ would almost always equal one, unless there was interest in understanding a more exact location of tags. In Chapter 3.2.2 an overlapping coverage network, square grid, proposes increasing alpha to better understand precise tag position.

This previous research in wireless communication can help to stimulate ideas of how to optimally cover space with readers and antennas, however these models assume the need for $100 \%$ coverage, which wireless communication customers expect from their products.

\section{2: Research in RFID Coverage Optimization}

Bryce Taylor (2008) proposed a method for optimizing the location of antennas to read tags at all times while minimizing the number of antennas in the network. He used principles of operations research such as the idea of making a continuous space into a discrete space by turning the facility into as grid, which was created by Francis et al. (1992) for facility location problems. The difficulty as Taylor describes it is "choosing the minimal number of points on the discrete grid such that the union of their 
corresponding subsets includes all the points on the grid (2008)." This resembles a minimum coverage problem that Garey and Johnson considered in 1979.

Taylor came up with both an optimal and a "greedy" solution to this problem. The optimal method, which can be burdensome and requires a lot of computing power for larger facilities, uses Microsoft Excel's Solver technology to generate a solution. The greedy method uses a heuristic algorithm proposed by Slavik in 1996 for minimum coverage, which chooses the covering set with the maximum elements left, deleting these elements from the remaining sets, and repeating until the space is completely covered. The difference between the upper and lower bounds of this data is less than 1.1 (Chvatal, 1979), proving that the algorithm results in a near optimal solution that can be generated much faster than in the Excel Solver.

Taylor looked to optimize the coverage of linear antennas mounted on walls to encompass situations where RFID tags travel at different heights from the readers, making the problem 3D as opposed to this paper, which considers tags traveling at a relatively equal height, allowing for 2D optimization. Taylor's research also focuses on complete coverage of the facility without considering if complete coverage is actually optimal based on the value of information provided by the RFID network.

\section{3: RFID Antenna Properties}

There are a number of factors that affect the read range of antennas that are important to consider before addressing the layout of the antennas. The first is the antenna gain, or power, that is used to activate passive tags or send the signal to identify the location of active tags. Antenna gains typically range anywhere from one to ten $\mathrm{dB}$ (Armstrong, 2013). For this paper, we will assume that there are antennas with enough 
power to read at a distance from their mounting point on the ceiling to the production height.

The polarization of the antenna also can impact the read rate of antennas and accuracy of the data collected. Linearly polarized antennas send out a beam that reads in a single plane; similar to a swing that can only travel back and forth. This type of antenna is often used at entryways, such as doorways, for tags that all have a similar orientation in relation to the antenna.

In contrast, circularly polarized antennas emit a signal in two planes that rotate around a single axis. These antennas' read ranges look similar to a hurricane on a Weather Doppler, where the tip of the storm projects from the antenna. Because power is split between two fields, circularly polarized antennas have shorter read ranges: however a more powerful antenna, or one placed closer to the working surface, can help to eliminate any read range issues (Armstrong, 2013).

For the systems discussed in this paper, circularly polarized antennas will be considered for their ability to read RFID tags in multiple orientations and the shape of the electromagnetic field. The circular shape of this field allowsone to calculate the radius and coverage area of the antenna by multiplying the distance from the antenna to the level of production by the sine of the angle projected. This angle is assumed to be 30 degrees in this paper.

There are other factors to consider when implementing antennas in a system such as energy lost to cords, the sizes and types of RFID tags, and environmental factors. For the purposes of this paper, we will consider these items negligible. 


\section{Chapter 3: Optimal Production Coverage Methods}

Given a rectangular area and circles with a known radius of $r$, the ideal minimum number of antennas in a network would be:

$$
N=\frac{\text { Area of the Rectangle }(X * Y)}{\text { Area of the Circle }\left(\pi r^{2}\right)}
$$

However this equation does not factor in the geometry of circles and their inability to completely cover a rectangular space without overlapping. The following sections will first consider two antenna networks that optimally cover a rectangular space without overlapping, and then two networks that optimally cover this same space while overlapping, essentially providing greater coverage with added implementation and maintenance costs.

\section{1: Optimal Coverage with Non-Overlapping Circles}

\subsection{1: X by Y Coverage}

The first method that is considered for antenna formation is when all of the antennas are placed in rows, where the centroid of the antenna's electromagnetic field, the location of the actual antenna, is lined up with other antennas on either side. Taking the area of a square surrounding the antenna's circular electromagnetic field and subtracting the area of this circle, it was found that $78.54 \%$ of space was covered. This assumes that the edges of each circle are touching.

The area of a square around a circle with a given radius $(r)$ is $2 r^{*} 2 r$ or $4 r^{\wedge} 2$. The area of the circle itself is $\mathrm{pi}^{*} \mathrm{r}^{\wedge} 2$. Dividing pi by four results in $78.54 \%$, which is the percent of coverage. 
To calculate the number of antennas in this system, divide the length of the facility by $2 \mathrm{r}$ and the same with the width. Multiplying these two numbers together will result in the total number of antennas in the system. If the length and width of the facility are divisible by $2 \mathrm{r}$, then the total space covered by the antennas is $78.54 \%$ of the area of the facility.

However, the dimensions of the facility are unlikely to be divisible by $2 \mathrm{r}$, and the remainder of this space on the edges of the formation is additional uncovered area. Another row of antennas can be added to cover this area, however this produces a less than optimal solution and will cost more. This uncovered space can be calculated using the equation below:

(Length of Facility $-2 \mathrm{r}\left(\mathrm{N}_{\mathrm{L}}\right)=$ Additional Uncovered Space $(\mathrm{L})$

Additional uncovered space is the length of the facility less the number of antennas in the long direction multiplied by $2 \mathrm{r}$. This same calculation can be made for width. The amount of uncovered space in a facility with a known length, width, and the radius of the antenna coverage is equal to:

$$
\left(.2146 \mathrm{r}^{\wedge} 2\right) \mathrm{N}+\left(\mathrm{L}-2 \mathrm{r}\left(\mathrm{N}_{\mathrm{L}}\right)\right)+\left(\mathrm{W}-2 \mathrm{r}\left(\mathrm{N}_{\mathrm{W}}\right)\right)
$$

Where $\mathrm{N}$ is the number of antennas, $\mathrm{N}_{\mathrm{L}}$ is number in the length direction, and $\mathrm{N}_{\mathrm{W}}$ is number in the width direction. 


\subsection{2: Hexagonal Lattice Coverage}

A more optimal method of covering an area without overlapping any circles is by placing the center of the circles in an equilateral triangle formation, creating a hexagonal lattice or honeycomb formation that covers $90.7 \%$ of space (Kershner, 1939). The image below shows the hexagonal lattice formation.

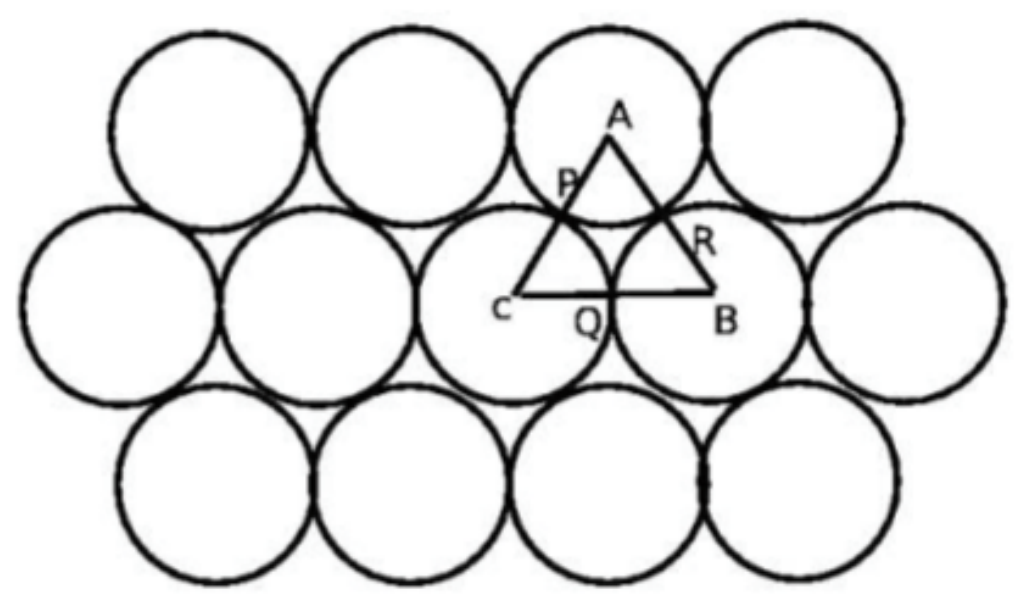

Figure 1: The Hexagonal Lattice Formation (Anusha, 2005)

The coverage can be calculated using the following equation:

$$
\begin{aligned}
\% \text { coverage }=\frac{\operatorname{Ar}(\text { sector } A P R)+\operatorname{Ar}(\text { sector } B Q R)+\operatorname{Ar}(\text { sector } C Q P)}{\operatorname{Ar}(\text { triangle } A B C)} \\
=\frac{3 x \frac{\pi r^{2}}{6}}{\sqrt{3 r^{2}}} \\
=.906899682
\end{aligned}
$$


Now that one knows the optimal coverage of non-overlapping circles, one must consider the importance or value of the information missing in the remaining $9.32 \%$ of space that cannot be covered in the hexagonal lattice formation.

\section{2: Optimization of Complete Floor Coverage Models}

Sometimes facilities need to know where their products are at all times, requiring complete coverage of the facility floor. Above, the idea of optimizing coverage with nonoverlapping circles produces a lower costing implementation due to fewer readers, however information can be lost in $9.32 \%$ of the facility. For complete coverage of a facility floor, a different optimal formation must be calculated. Of course these proposed overlapping systems will require more antennas that in turn will increase the cost of implementation; however the value of this additional information may be worth the upfront costs.

\subsection{1: Optimal Overlapping of Circles}

In 1938 Richard Kershner created a theorem that stated that for a bounded plane $\left(\mathrm{X}^{*} \mathrm{Y}\right)$, the minimum number of circles of radius $\mathrm{r}$ can be calculated using the following equation (Anusha, 2005):

$$
N_{\text {optimal }}=\left(\frac{2 \sqrt{3 X Y}}{9 r^{2}}\right)
$$

The density of an optimal layout, the ratio of total area covered by the sum of the area of the circles and the total area to be covered, is equal to 1.209.

$$
\frac{(2 \pi \sqrt{3})}{9}=1.209
$$




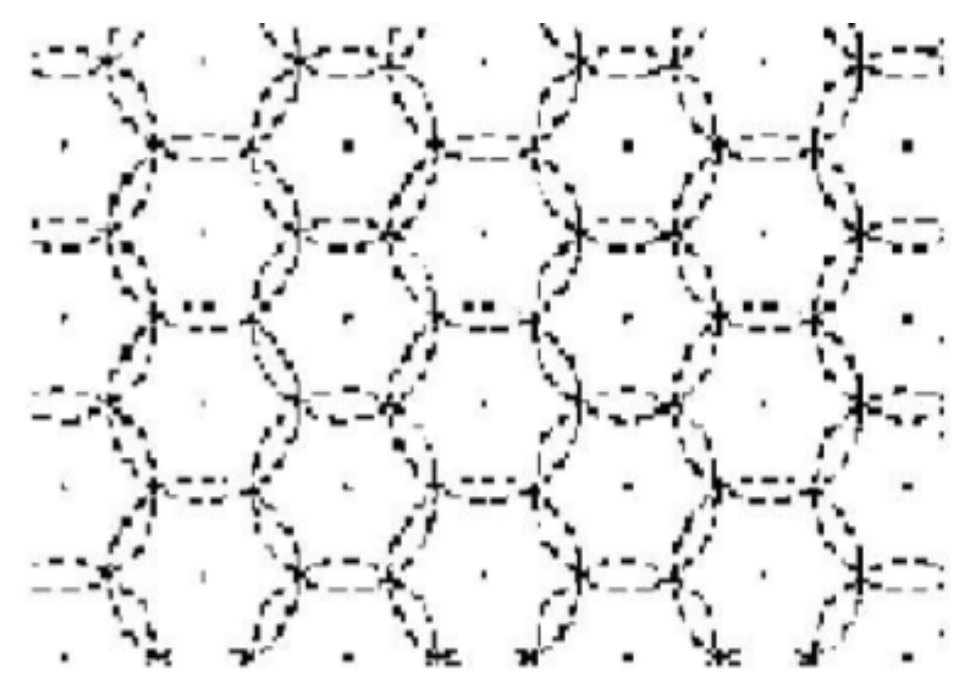

Figure 2: $120.9 \%$ area of the circles is the minimum area required to cover the entire space (Anusha, 2005)

\subsection{2: Square Grid RFID Reader Antenna Network}

A concern with the methods described above is the uncovered space that tags could potentially be located in with no ability to be tracked, as well as a system that returns a wide area that the tag could fall into. Even with the $90.7 \%$ coverage of the hexagonal lattice, there are other issues when trying to pinpoint the exact location of the items. Because there are no overlapping electromagnetic fields, tags read by a particular antenna can show a general area of where the product is located. This area is equal to the $\operatorname{pi}\left(\mathrm{r}^{\wedge} 2\right)$ or the area of the antenna's magnetic field.

To gain a greater insight into the exact location of the tagged products and to cover nearly one hundred percent of the production floor, a formation of antennas known as the "Square Grid RFID Antenna Network" can be implemented. The layout of this network is pictured below. The bolded points represent the locations of the reader antennas, and the circles, each with a radium or $r$, show the coverage of these readers.

This network presents the optimal coverage of a vast majority of the production facility, with the area inside the grid zone being $100 \%$ covered. Areas without $100 \%$ 
coverage are the areas in the non-grid zone, or the walls of a facility, as the other methods have reflected this same issue when looking to optimally cover the entire facility.

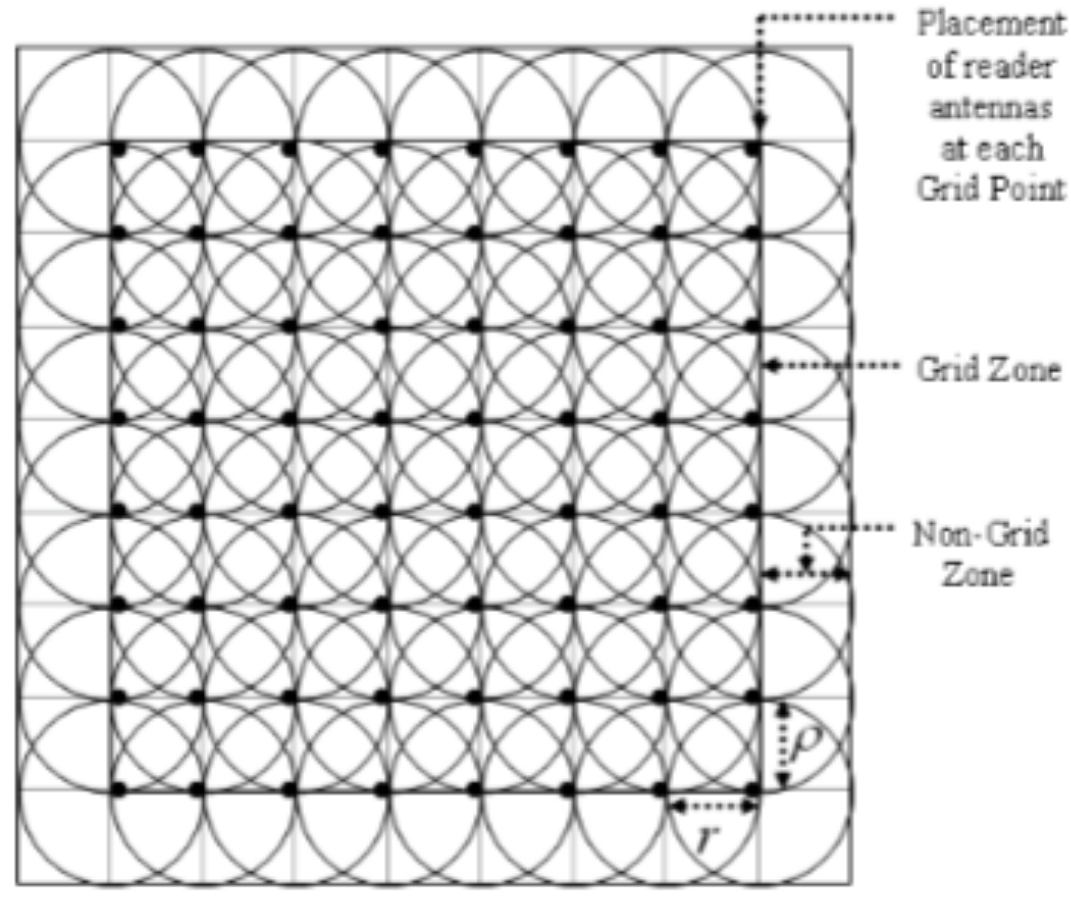

Figure 3: Square Grid Method Sample Layout (Tan et al., 2008)

For a rectangular facility length $\mathrm{A} \times \mathrm{B}, \mathrm{A} / \mathrm{r}=\mathrm{L}$ and $\mathrm{B} / \mathrm{r}=\mathrm{W}$, where $(\mathrm{L}-1)$ is equal to the required number of gridlines in the length direction, and (W-1) is the required number of gridlines in the width direction (Tan et al., 2008); the required number of antennas for the whole system is simply $(\mathrm{L}-1)^{*}(\mathrm{~W}-1)$. This number is nearly double that of the simple model created above, while only covering a slightly larger area.

Though this would lead to a sizable increase in upfront cost from the previous models, there is another benefit of the square grid network and that is its ability to more precisely locate tagged items in a system. By using a diffusion algorithm, one can use the overlapping circles to estimate the position of the tag. Looking at a basic section of four 
overlapping circles (Figure 4), four distinct patterns can be seen. With more overlapping comes smaller areas, and as a result, a more specific tag location.

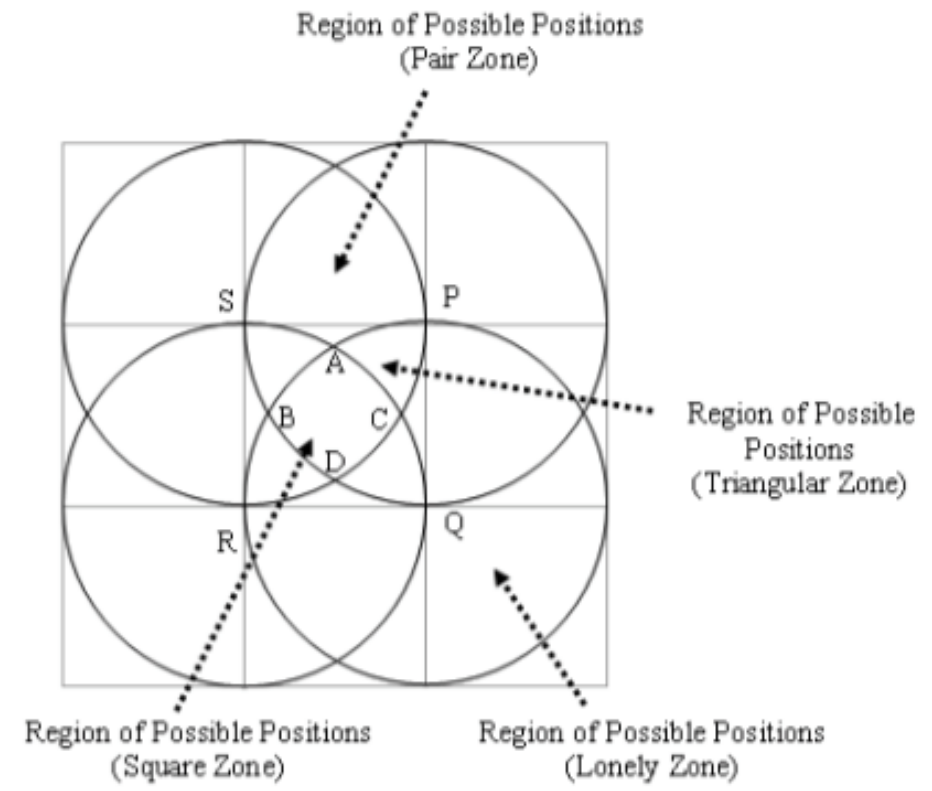

Figure 4: Four Overlapping Sections in Square Grid

A study was conducted where $10 \%$ of tags were placed in a lonely zone, $30 \%$ of tags in a pair zone, $6 \%$ of tags in a triangular zone, and $24 \%$ in the square zone. The purpose was to understand the difference between the estimated location of the tags and the actual location. The results showed that the mean distance from the tag's estimated location was .26 meters, while the variance was .06 meters.

This method was compared against two other location-tracking options, Wi-Fi and BlueBot, and it was found to be far superior. With two different experiment sets, each with varying location of tag placement, the square grid had an average error of $.3525 \mathrm{~m}$ compared with $1.4839 \mathrm{~m}$ using BlueBot and $4.0916 \mathrm{~m}$ using Wi-Fi in the first set, and $.1935 \mathrm{~m}$ versus $1.0521 \mathrm{~m}$ and $2.6571 \mathrm{~m}$ in respective sets. These results show the 
superiority of the RFID square method over other forms of industry accepted location technology, however this accuracy comes at an additional cost compared to some of the other models that have been previously evaluated (Tan et al., 2008). 


\section{Chapter 4: The Value of Information}

The value of a real-time location tracking system is its ability to provide a manufacturing facility management team with more data. This information in turn allows for a better understanding of the production process and can highlight areas where improvements can be made. These systems can also help locate particular items such as tools or goods that are being produced for all sorts of functions.

With an understanding of potential optimal layouts and associated implementation costs referenced in chapter three, we can look to access and quantify the value of information based on coverage and determine the optimal RFID system layout. The goal of this chapter is to analyze the value of information versus the additional implementation costs associated with adding more antennas into the system.

\section{1: Method of Quantifying the Value of Information}

Whatever the need, a real-time location tracking system provides information that's value is equal to the difference between an expected outcome when a decisionmaker has this information versus the expected outcome when there is no information provided by the system (Lawrence, 1999). This is based on the economic idea of decision theory or "statistical theory concerned with quantifying the process of making choices between alternatives" (Merriam-Webster, 2015).

By creating a probability distribution to represent a decision maker's belief of an item's location in a particular space before receiving any information, and comparing that to the distribution following the receipt of information from the RFID system, also known as the posterior distribution, one can begin to calculate the value of the RFID system. These distributions help to calculate the amount of saved timed for a decision 
maker with and without information, which can then be converted to cost based on a company's cost of labor. This calculation reflects the normative value of information, or the average value expected to deliver to the decision maker in the long term (Kelepouris \& McFarlane, 2010). There is also the actual value of information, specific to particular instances within the system rather than the system as a whole; however the goal of this paper is to come up with a general equation to implement in any system.

\subsubsection{Measurement Method}

The measurement of value provided by the RFID system is broken into three major steps. The first is location estimation, where one is able to formulate both the prior and posterior distributions and use the information to indicate the role of information in improving the estimation of location. The second step breaks down the possible action steps for a decision maker. These are important because the more possible actions for an uninformed decision maker can lead to high value provided by the RFID system. The third and final step is where the expected posterior payoff and expected prior payoffs are calculated and compared. The following sections will further break down each of these steps and will show how one can calculate the value of their RFID system and its coverage. A visual representation of this can be seen in the figure below. 


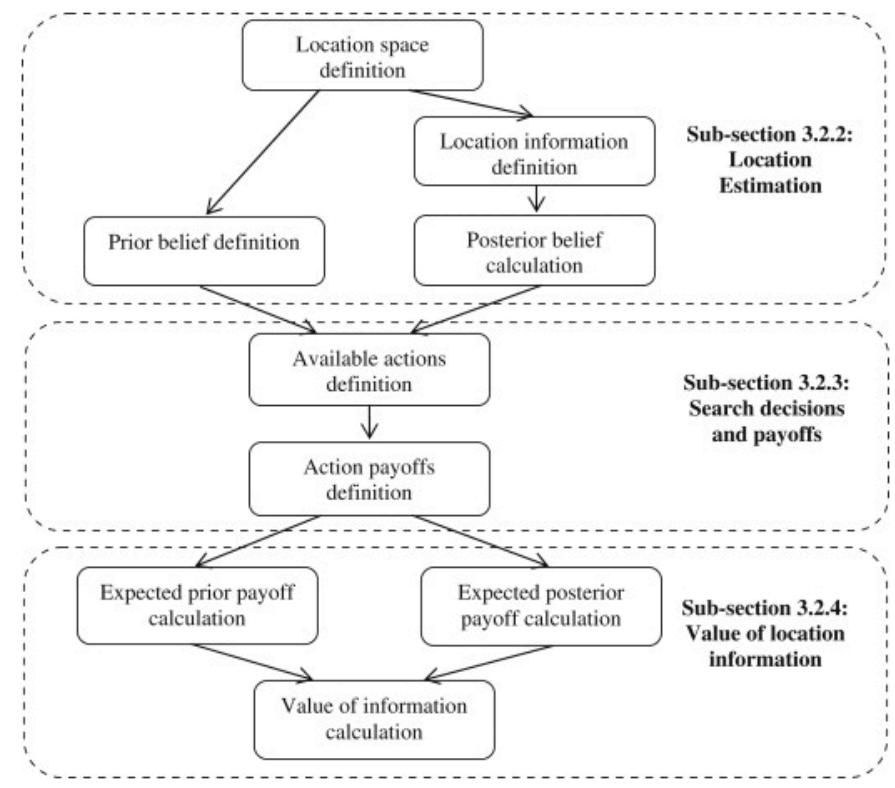

Figure 5: Accessing the Value of Information (Kelepouris \& McFarlane, 2010)

\subsubsection{1: Location Estimation}

In the location estimation step of the measurement model, one must first understand the two spaces considered. The first space is the state space, which can be described as subsections of the entire facility. Each state should require equal search time for a decision maker seeking an object if they are standing in the middle of that state. The more equal these state spaces are in terms of search time, the more accurate the final calculation will be. However, there is a trade-off between the measurement complexity and accuracy of the results. As the number of state spaces increases, the more complex the final computation becomes (Kelepouris \& McFarlane, 2010).

The second space that will be considered is signal space. This is the space in which information can be received, or the coverage of the RFID system. D.B. Lawrence (1999) proves that making the signal space more granular than the state space does not add value to the decision-making processes, and in turn will not improve the accuracy of 
the measurement. This is very important to consider when the different optimization methods are discussed later, particularly when considering the Square Grid Network, which most likely would provide more granularity than necessary. For increased precision, one would have to increase the number of state spaces, allowing for an increase in signal spaces: however this would add further complexity to the calculation as mentioned above.

For the prior distribution only the state space is considered. It can be assumed that the decision maker has a belief about the "likely" location of an object that can be represented with the probability distribution over the state space $P(Z)$ such that:

$$
\sum_{i=1}^{N_{Z}} p\left(z^{i}\right)=1
$$

Where $\mathrm{Z}$ is the number of state spaces, and $\mathrm{p}\left(z^{i}\right)$ is the probability that the object is in state $z^{i}$. This information can be collected from process experts who can offer an estimate of the typical location of an object based on this object's use. The academic means of populating a distribution using expert knowledge can be found in D.B. Lawrence's book The Economic Value of Information (1999). Another option for populating the prior distribution is by recording an object location over time to capture the object's usual behavior (Kelepouris \& McFarlane, 2010).

Creating the posterior distribution includes information about both the signal space $(\mathrm{Y})$ and state space $(\mathrm{Z})$. The accuracy of the location signals based on the objects real location can be represented by a conditional probability distribution in the form of a

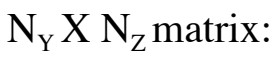




$$
P(Y \mid Z)=\left[\begin{array}{ccc}
p\left(y_{1} \mid z^{1}\right) & \cdots & p\left(y_{N_{Y}} \mid z^{1}\right) \\
\vdots & p\left(y_{j} \mid z^{i}\right) & \vdots \\
p\left(y_{1} \mid z^{N_{2}}\right) & \cdots & p\left(y_{N_{Y}} \mid z^{N_{2}}\right)
\end{array}\right]
$$

The ijth element is the probability of receiving signal $y_{j}$ when the object is actually in the state $\mathrm{z}^{\mathrm{i}}$. This matrix is row-stochastic meaning that $\sum_{j=1}^{N_{Y}} p\left(y_{j} \mid z^{i}\right)=1$. This states that the sum of the probabilities of an object being in the state where the object is seen by the signal space is equal to one. By multiplying $\mathrm{P}(\mathrm{YIZ})$ by $\mathrm{P}(\mathrm{Z})$, which is used for the prior probability, one can calculate the likelihood of receiving each signal $\mathrm{y}_{\mathrm{j}}$ (Kelepouris \& McFarlane, 2010).

$$
p\left(y_{j}\right)=\sum_{i=1}^{N_{Z}} p\left(y_{j} \mid z^{i}\right) p\left(z^{i}\right)
$$

The next step is to calculate a posterior belief about the location of the object or $\mathrm{P}(\mathrm{ZIY})$, which is expressed $\mathrm{N}_{\mathrm{z}} \mathrm{X} \mathrm{N}_{\mathrm{y}}$ matrix below:

$$
P(Z \mid Y)=\left[\begin{array}{ccc}
p\left(z^{1} \mid y_{1}\right) & \cdots & p\left(z^{1} \mid y_{N_{Y}}\right) \\
\vdots & p\left(z^{i} \mid y_{j}\right) & \vdots \\
p\left(z^{N_{2}} \mid y_{1}\right) & \cdots & p\left(z^{N_{Z}} \mid y_{N_{Y}}\right)
\end{array}\right]
$$

The ijth element, which is the element we are interested in using as a comparison to the prior can be calculated using Bayes' rule:

$$
p\left(z^{i} \mid y_{j}\right)=\frac{p\left(y_{j} \mid z^{i}\right) p\left(z^{i}\right)}{p\left(y_{j}\right)}
$$


This equation calculates the probability of an item in $z^{i}$ based on $y_{j}$ by taking the likelihood of receiving $\mathrm{y}_{\mathrm{j}}$, as found above, and dividing that by the probability of signal state $y_{j}$.

By comparing $p\left(z^{i} \mid y_{j}\right)$ or the posterior with $p\left(z^{i}\right)$ or the prior, one can begin to see the value of information in improving the estimation of an object's location.

\subsubsection{2: Search Decisions Based on Available Actions}

To find an object, one must select a path of actions that will be followed until that object is found. This search path, or set of available actions $a_{j}$, consists of a sequence of all the location states $a_{j}=z^{a} \rightarrow z^{b} \rightarrow \cdots \rightarrow z^{N} z$ in which the decision maker anticipates visiting until the object has been located. It can be assumed that the decision maker does not search the same space twice, and once the object is found the search stops. This leaves the opportunity for $\mathrm{N}_{\mathrm{Z}}$ ! possible paths of action; however a number of these paths can be reduced because there are some paths that would never occur, such as a potentially allergenic product outside of it's designated location. The set of all meaningful paths a decision maker may take makes up the set of available actions $A=\left\{a_{j} ; i=1, \ldots, N_{A}\right\}$ (Kelepouris \& McFarlane, 2010).

The aim of the decision maker is to select the path that gets him the object as quickly as possible, essentially minimizing the amount of time spent searching in wrong locations. The aim would be to start one's path of action in the state space where the item is located because each action in the path requires a certain amount of search time. The sum of these search times defines the payoff, which in this case is negative because the goal is to have to shortest search time possible, reducing the overall cost. The total time spent searching for the object or payoff will be $\omega\left(z^{i}, a_{k}\right)$, which is comprised of all the 
search times plus travel times between state spaces. Walking time between space states a and $\mathrm{b}$ is represented by $t_{z_{a} \rightarrow z_{b}}^{w}$, while the search time in state $\mathrm{b}$ is represented by $t_{z_{b}}^{S}$. The action path, $\mathrm{a}_{\mathrm{k}}$ with the object being in state $\mathrm{z}^{\mathrm{i}}$ is represented by $a_{k}=z^{a} \rightarrow z^{b} \rightarrow \cdots \rightarrow$ $z^{i}$. The resultant is:

$$
\omega\left(z^{i}, a_{k}\right)=t_{Z_{a}}^{S}+t_{Z_{a} \rightarrow Z_{b}}^{w}+t_{Z_{b}}^{S}+t_{Z_{b} \rightarrow Z_{c}}^{w}+\cdots+t_{Z_{i-1} \rightarrow Z_{i}}^{w}+t_{Z_{i}}^{S}
$$

(Kelepouris \& McFarlane, 2010)

These times can all be easily measured and can be placed into a payoff matrix that helps to specify a payoff between a specific action path and the object state.

$$
\omega(z, a)=\left[\begin{array}{ccc}
\omega\left(z^{1}, a_{1}\right) & \cdots & \omega\left(z^{1}, a_{N_{A}}\right) \\
\vdots & \omega\left(z^{i}, a_{k}\right) & \vdots \\
\omega\left(z^{N_{z}}, a_{1}\right) & \cdots & \omega\left(z^{N_{z}}, a_{N_{A}}\right)
\end{array}\right]
$$

\subsubsection{3: Calculating The Value of Information}

By combining the formulations discussed in the previous two sections, one can begin to calculate the actual value of information. The first step is to calculate the expected prior payoff, which is the sum of all possible payoffs multiplied by the probability of the object being in that state.

$$
\bar{\Omega}\left(\omega, a_{k}\right)=\sum_{i=1}^{N_{Z}} \omega\left(z^{i}, a_{k}\right) p\left(z^{i}\right)
$$

(Kelepouris \& McFarlane, 2010)

Based on the expected utility axiom, which states that a decision maker will do anything they can to maximize benefits (von Neumann \& Morgenstern, 1947), the searchers will attempt to find the object as quickly as possible. 


$$
a^{*}=\operatorname{argmax} \sum_{i=1}^{N_{Z}} \omega\left(z^{i}, a\right) p\left(z^{i}\right)
$$

With no information available, the decision maker will choose their initial action $\mathrm{a}^{*}=\mathrm{a}_{0}$ based on their belief of the location that maximizes their payoff based on the distribution of $\mathrm{P}(\mathrm{Z})$. The expected prior payoff is represented by:

$$
\bar{\Omega}\left(\omega, a_{0}\right)=\sum_{i=1}^{N_{Z}} \omega\left(z^{i}, a_{0}\right) p\left(z^{i}\right)
$$

This equation can calculate the expected time it will take a decision maker to find this object when there is no location information available.

For the posterior calculation, the action path is now maximized by the information received from the signal state $y_{j}$, from which the decision-maker will choose the action $a_{y j}$ that maximizes the payoff:

$$
a_{y_{j}}=a \operatorname{gmax} \sum_{i=1}^{N_{Z}} \omega\left(z^{i}, a\right) p\left(z^{i} \mid y_{j}\right)
$$

The posterior expected payoff is the sum of the expected payoff when receiving each signal of $y_{j}$ multiplied by the likelihood or receiving that signal.

$$
\bar{\Omega}(\omega, A \mid Y)=\sum_{j=1}^{N_{Z}}\left[p\left(y_{j}\right) \sum_{i=1}^{N_{Z}} \omega\left(z^{i}, a_{y_{j}}\right) p\left(z^{i} \mid y_{j}\right)\right]
$$

This equation represents the expected time a decision-maker will spend searching for an object using information from the RFID system. 
With these two payoff formulas, one can calculate the value of information:

$$
\begin{gathered}
\operatorname{VOI}(\omega, A, Y)=\bar{\Omega}(\omega, A \mid Y)-\bar{\Omega}\left(\omega, a_{0}\right) \\
=\sum_{j=1}^{N_{Z}}\left[p\left(y_{j}\right) \sum_{i=1}^{N_{Z}} \omega\left(z^{i}, a_{y_{j}}\right) p\left(z^{i} \mid y_{j}\right)\right]-\sum_{i=1}^{N_{Z}} \omega\left(z^{i}, a_{0}\right) p\left(z^{i}\right)
\end{gathered}
$$

Now what needs to be looked at is how this value of information equation can be applied to the idea of optimizing the RFID coverage of a manufacturing floor. In this next section, the idea of inaccuracy due to an uncovered area will be discussed to come to a conclusion on the optimal organization of RFID readers.

\subsection{2: Determining the Impact of Missing Information}

An experiment was conducted to quantify the time savings attributed to the proposed value of information equation and to identify the benefits affected by the existence of the blind spots where reliable location information is not available. This second objective is crucial in understanding the benefits of an optimal overlapping system that covers the entire facility floor versus the hexagonal lattice layout what only covers $90.7 \%$ of space.

As stated in the section above, there is no additional value added by creating signal spaces that are more granular than the state space to the decision-making processes because it will not improve the accuracy of the measurement. Due to this factor, it will be assumed that each state space is equal to the signal space, or the area of the RFID antennas projected radius on the facility floor. 
The $10^{\text {th }}$ state space in the experiment is any area not covered by these antennas, also know as a "blind spot" or "information inaccuracy" in the system. The probability of an item being in the blind spot in the system can be represented by the following equation:

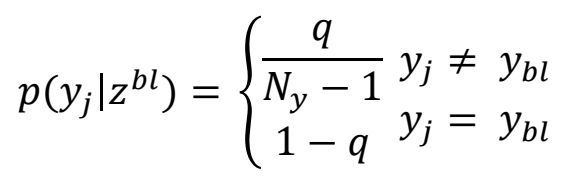

where $\mathrm{q}$ is equal to the percent of information inaccuracy. The other term used to help create their sensitivity analysis was "uncertainty," which is considered in the prior distribution and is expressed as a percentage of noise, distributed among the states. This percentage reflects the lack on knowledge of an item's location one would have if no system existed. The figure below shows an example of how uncertainty can be distributed:

\begin{tabular}{rcccccccccc}
\hline State tool & 1 & 2 & 3 & 4 & 5 & 6 & 7 & 8 & 9 & 10 \\
\hline Tool 1 (class 1) & $18 \%$ & $18 \%$ & $18 \%$ & $18 \%$ & $2 \%$ & $2 \%$ & $18 \%$ & $2 \%$ & $2 \%$ & $2 \%$ \\
\hline
\end{tabular}

Figure 6: Distribution of Uncertainty (Kelepouris \& McFarlane, 2010)

The revised prior distribution under initial uncertainty can be defined by the following function:

$$
p^{u}\left(z^{i}\right)= \begin{cases}p\left(z^{i}\right)-\frac{u}{N_{p\left(z^{i}\right) \neq 0}}+\frac{u}{N_{z}}, & p\left(z^{i}\right) \neq 0 \\ u / N_{z}, & p\left(z^{i}\right) \neq 0\end{cases}
$$

Where $N_{p\left(z^{i}\right) \neq 0}$ is the number of non-zero states.

Examining the sensitivity analysis performed on the RTLS system seen in the figure below, it was concluded that the increase in information accuracy resulted in "only 
marginal increase in savings, assuming that the initial uncertainty is kept the same.

(Kelepouris \& McFarlane, 2010)" However, it was also found that the increase in initial uncertainty leads to a significant rise in time savings.

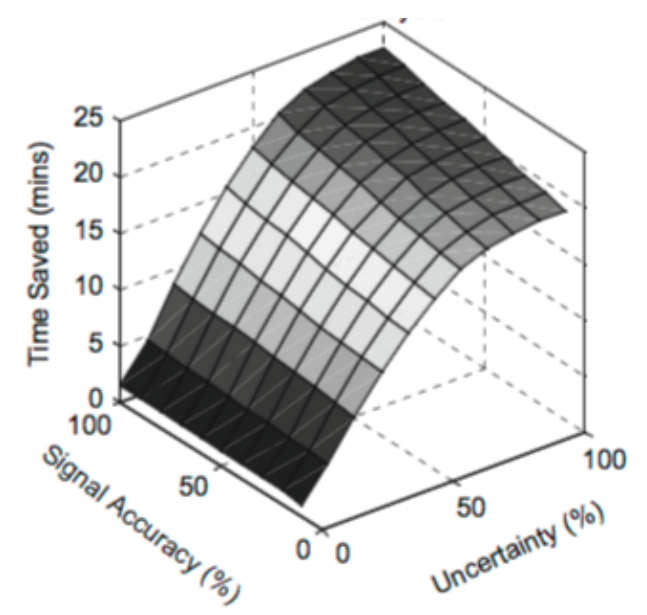

Figure 7: Sensitivity Analysis of RTLS

By looking at the downward slope from the y-axis towards the axis labeled "Uncertainty (\%)," one can see that the signal accuracy makes limited impact on the time savings in the experiment conducted. This savings is what one would look for to justify the additional upfront costs of system implementation of the more expensive optimal overlapping layout.

These equations proposed above as well as high knowledge of one's current processes and procedure can help one to calculate the value of information in their system. However, this finding of limited benefit from an additional increase in accuracy information leads one towards implementing the low cost hexagonal layout. These two systems will be evaluated on these parameters in the next section. 


\subsection{3: Analysis Based on Quantitative Model}

To this point, this paper has covered four possible optimal layouts for covering a production facility. Each layout has its advantages, however the ones analyzed in this paper cover only the costs of implementation based on the number of antennas one would need to purchase versus the added value of information that additional antenna would bring.

Two of these solutions were eliminated: one due to a lack of optimization, which lead to lower area coverage, and the second due to an overabundance of non-useful information the layout provided by its redundancy in coverage. This leaves one to consider the differences between the hexagonal lattice layout, the optimal nonoverlapping layout, and the optimal overlapping layout.

The hexagonal lattice layout covers $90.7 \%$ of space, while requiring $\mathrm{N}_{h}$ antennas, calculated using the following equation:

$$
N_{h}=\left(\left(F_{L} * F_{W}\right) * .907\right) / \pi r^{2}
$$

where $F_{L}$ equals factory length and $F_{W}$ equals factory width. This simplified equation does not include additional space along the walls that could be covered with an additional row of antennas that cover this area without fully efficient coverage, covered with wall mounted RFID antennas, or left as blind spot due do a lack of activity or production in the facility.

In comparison, the optimal overlapping layout requires:

$$
N_{o}=\left(\left(F_{L} * F_{W}\right) * 1.209\right) / \pi r^{2}
$$


This system requires $34 \%$ more antennas that the hexagonal lattice formation as can be seen in the equation below.

$$
\left(\frac{1.209-.907}{.907}\right) * 100=33.3 \%
$$

This can be represented by $\mathrm{N}_{\mathrm{h}}=1.333 \mathrm{~N}_{\mathrm{o}}$. For a small system with few antennas, this discrepancy has minimal impact. However for a larger system, the cost of implementation begins to increase rapidly.

Let it be assumed that one is looking to cover a rectangular factory that is $100 \mathrm{ft}$. long and $250 \mathrm{ft}$. wide. The RFID antennas are going to be mounted $25 \mathrm{ft}$. from the level of the RFID tags, assumed to be a production line. With the assumption that the RFID antenna projects down at an angle of 30 degrees, the radius of the circles will be $25 / \sqrt{3}$ $14.43 \mathrm{ft}$., which for this simple equation we will assume to be 15 feet. The picture below shows how this value was calculated for a 30-60-90 triangle (CK-12 Foundation, 2016).

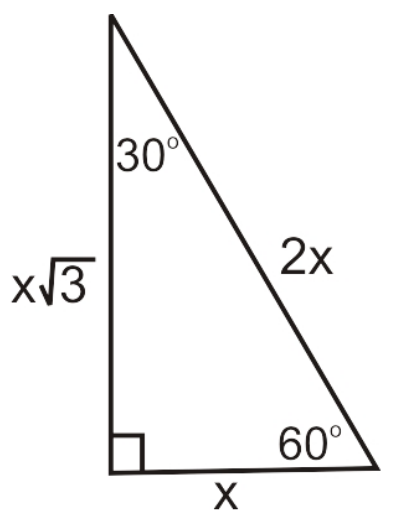

Figure 8: 30-60-90 Triangle

For the hexagonal lattice formation, we can now take the area of the facility and multiply that number by $90.7 \%$, then divide this area by the area of our antenna's projection.

$$
((100 * 500) * .907) / \pi 15^{2} \approx 64 \text { Antennas }
$$


For the optimal overlapping solution we can multiply this number by 1.333 , however to confirm our equation, we will calculate the number of required antennas. To do this once again, we will take the area of the facility and multiply it by 1.209 and then divide it by the area of the circle.

$$
((100 * 500) * 1.209) / \pi 15^{2} \approx 86 \text { Antennas }
$$

This method results in an additional 22 antennas. Each additional antenna ranges in price from $\$ 100$ and $\$ 500$, while each additional receiver for every four antennas costs between $\$ 1,000$ and $\$ 5,000$ (Taylor, 2008). Assuming the cost of antennas to be $\$ 300$ and the cost per receiver to be $\$ 3,000$, the additional implementation costs, without including the cost of the physical installation or additional maintenance costs, comes out to an additional $\$ 25,000$.

\section{2: Value of Information}

Kelepouris and McFarlane (2010) concluded that an incremental increase in information accuracy leads to very limited added value in information for a dollar perspective. The sensitivity analysis from their experiment showed that the minutes saved per day were almost identical for a system with $90 \%$ accuracy versus a system with $100 \%$ accuracy. Even if that difference were 2 minutes a day, an individual could save 12 hours per year.

Assuming a salary of $\$ 25 /$ hour, it would take one year to break even on the hardware costs for each additional antenna in the system. In the example above, it would take around 80 years to get a payback for the additional antennas required to cover $100 \%$ of the facility floor instead of simply $90 \%$. This being said, based on this quantitative 
approach, the optimal solution for maximizing information while minimizing implementation costs is the hexagonal lattice formation.

Thus far, only a quantitative analysis has been considered. However, there are other factors that can have an impact on management's decision about what system to implement. Some facility managers may be willing to pay higher upfront system costs to have greater visibility into their process. They could potentially leverage a system that completely covers a production floor to collect data on their process.

With a good experiment design, an Industrial Engineer (IE) could look at the output data points for the RFID system for numbers that could be statistically evaluated to make improvements on the system. This could save a company hundreds of dollars in data collection and allow IEs to better pinpoint issues in the system. Though this could be done with certain outputs in a warehouse with $90 \%$ coverage; the ability to collect data may be eliminated if the coverage is not in the right spot. .

Another advantage to being able to track the exact location of goods comes when dealing with products of very high value. Losing track of these goods in production could lead to excess scrap or product loss, resulting in negative impacts to the bottom line.

Also, items that can be tampered with could also require extra tracking. The system could be configured to alarm when a good does not follow the normal rate through production, notifying management of any possible issues with products.

Though these are very valid considerations, it is challenging to access the value that this added information would provide. There isn't really any literature available on these costs because they are different for each facility based on the product and the way decision makers leverage information available to them. On top of that, it is really 
challenging to quantify additional savings that could be provided with perfect information versus $90 \%$ of information. 


\section{Chapter 5: Conclusion}

The problem with optimizing the coverage of a facility floor with RFID technology was addressed through the analysis of four different networks for laying out RFID antennas, both non-overlapping and overlapping options. For each of these two categories, the superior network based on optimization and the value that network provides was selected. The optimal non-overlapping network was concluded to be the hexagonal lattice formation, a formation of circles seen many times in nature, such as the formation of water molecules in their solid state, and proven to be optimal in the coverage of space by Kershner in 1938. The optimal overlapping network was also proposed by Kershner and states that the overlapping circles total area is equal to 1.209 times the area of the space that the circles cover.

The optimal non-overlapping method covers $90.3 \%$ of space, while the optimal overlapping model can cover $100 \%$ of the facility, however this comes at an additional cost. The cost of implementation for the optimal overlapping method comes with an additional $34 \%$ in comparison to the non-overlapping method and only provides information on $9.7 \%$ of space.

From a quantitative perspective this RFID system would struggle to pay for itself sheerly based on its added efficiency. Based on a study by Kelepouris et. al, a sensitivity analysis showed approximately $4 \%$ added value for a network covering $100 \%$ of space versus that which covers $90 \%$ of the facility. When spending an additional $34 \%$ on a system, $4 \%$ added value would often not pay for itself.

However, there are other considerations that a management team must deliberate upon such as the added value of knowing information $100 \%$ of the time, whether 
products need to be kept at certain temperature throughout production, or the financial impact if expensive items are missing in the system. These situations may require the overlapping optimal coverage of the facility.

Often there is a desire to have as much information as possible at all times, however this added information sometimes comes at a cost that is not justified by the benefits it provides.

This compilation of ideas, theories, and experiments has helped to provide clarity for a management team looking to implement an RFID system in their facility and has shown that the hexagonal lattice formation non-overlapping network provides the most optimal results based on costs of system implementation and value provided by the system. However, it leaves opportunity for certain production facilities to implement the optimal overlapping network based on their individual needs and requirements. 


\section{WORKS CITED}

Adickes, M., Billo, R., Norman, B., Banerjee, S., Nnaji, B., \& Rejgopal, J. (2002). Optimization of indoor wireless communication newowrk layouts. IIR Transactions , 823-826.

Anusha, S. (2005). RFIDcover - A Coverage Planning Tool for an RFID Network with Mobile Readers. Masters Thesis, Indian Institute of Technology Bombay.

Armstrong, S. (2013, July 1). 6 Factors that Affect RFID Read Range. Retrieved January 21, 2016, from RFID Insider.

Chvatal, V. (1979). A greedy heuristic for the set covering problem. Mathematics of Operations Research , 233-235.

CK-12 Foundation. (2016). Relationships of Sides in 30-60-90 Right Triangles. Retrieved March 6, 2016, from cK-12.

Dobkin, D. (2007). The RF in RFID. Philadelphia, PA: Elsevier .

Francis, R., McGinnis, L., \& White, J. (1992). Facility Layout and Location: An Analytical Appraoch. Englewood Cliffs, NJ: Prentice Hall.

Garey, M., \& Johnson, D. (1979). Computer and Intractability: A Guide to the Theory of NP-Completeness. Freeman.

Huang, C., Tseng, Y., \& Lo, L. (2004). The coverage problem in three dimensional wireless sensor networks. IEEE Globlecom, (pp. 3182-3186). Dallas.

Kelepouris, T., \& McFarlane, D. (2010). Determining the Value of Asset Location Information Syatems in a Manufactruing Environment. International Journal of Production Economics , 324-334.

Kershner, R. (1939). The Number of Circles Covering a Set. American Journal of 
Mathematics , 61 (3), 665-671.

Lawrence, D. (1999). The Economic Value of Information. New York, New York: Springer New York.

Merriam-Webster. (2015). Decision Theory. Retrieved from Merriam-Webster.

Omni-ID. (2010, March 17). Retrieved January 15, 2016

Patil, A., Munson, J., Wood, D., \& Cole, A. (2008). Bluebot: Asset Tracking Vie Robotic Location Crawling. Computer Communications , 31 (6), 1067-1077.

Slavik, P. (1996). A tight analysis of the greedy algorithm for set cover. 28th ACM Symposium on Theory of computing, (pp. 435-441).

Tan, K. G., Wasif, A. R., \& Tan, C. P. (2008). Objects Tracking Utilizing Square Crid RFID Reader Antenna Network. Journal of Electromagnetic Waves \& Applications , 22 (1), 27-38.

Taylor, B. (2008). RFID Antenna Coverage Optimization. California Polytechnic State University, Industrial Engineering.

Violino, B. (2005, June 16). What is RFID? Retrieved January 8, 2016, from RFID Journal.

von Neumann, J., \& Morgenstern, O. (1947). Theory of Games and Economic Behavior. Princeton: Princeton University Press. 